\title{
Variability in Anesthesia Models of Care In Cardiac Surgery
}

\author{
Dianne McCallister ${ }^{1}$, Bethany Malone ${ }^{2}$, Jennifer Hanna ${ }^{3}$, and Michael S Firstenberg 3,* \\ 1 Diagnosis Well, Inc, Greenwood Village, Colorado, USA; diannemcca12@gmail.com. \\ 2 Department of Surgery, Summa Akron City Hospital, Akron, Ohio, USA; Bcbmalone@gmail.com. \\ 3 Department of Cardiothoracic and Vascular Surgery, The Medical Center of Aurora, Aurora, Colorado, \\ USA; msfirst@gmail.com. \\ * Correspondence: msfirst@gmail.com
}

\begin{abstract}
The operating room in a cardiothoracic surgical case is a complex environment, with multiple handoffs often required by staffing changes, and can be variable from program to program. This study was done to characterize what types of practitioners provide anesthesia during cardiac operations to determine the variability in this aspect of care. A survey was sent out via a list serve of members of the cardiac surgical team. Responses from 40 programs from a variety of countries showed variability across every dimension requested of the cardiac anesthesia team. Given that anesthesia is proven to have influence on the outcome of cardiac procedures, this study indicates the opportunity to further study how this variability influences outcomes, and to identify best practices.
\end{abstract}

Keywords: Cardiothoracic Surgery; Anesthesia Staffing Models; Outcomes; Operating Room Staffing; Handoffs; Quality; Communication

\section{Introduction}

The operating room is a complex environment, with multiple team members, many of whom may move on and off the team based on shifts and other factors. The use of handoffs is a common procedure that can be employed to try to create continuity of care. In major operations, handoffs between anesthesia providers have been associated with increased mortality rates, readmissions, and major complications [1]. However, few studies address the diversity which includes which how many, and what training, of providers are actually providing anesthesia or other necessary intraoperative services nor the impact that such diversity has on clinical outcomes. The aim of this study is to characterize what types of practitioners provide anesthesia services during cardiac operations. We hypothesize that there exists a large variability in the composition, structure, and functioning of the Anesthesia providing team in cardiac surgery across programs.

\section{Materials and Methods}

An anonymous online survey was sent out to international cardiac surgery programs. No statistical analysis was performed due to the low number of responses and the large variability in responses - a finding that helps support the hypothesis that there exists a large variability in how (and by whom) cardiac surgical patients are managed in the operating room.

\section{Results}

The survey was distributed via a closed-membership international "listserv" Internet-based communication forum (OpenHeart-L@lists.hsforum.com), an international community of 
cardiothoracic surgeons, related providers (i.e. cardiac anesthesiologist, nursing, etc), representatives of industry, and interested members of the lay community. It is estimated that the Forum consists of over 1,000 individual members. Voluntary responses were obtained from 40 programs. The distribution in program sizes and responses is illustrated in Table 1. For smaller programs ( $<200$ cases/year), 38\% have certified registered nurse anesthetists (CRNA) managing cardiac patients with $62 \%$ having only physician anesthesiologists involved in the intra-operative management. Likewise, for medium programs (201-749 cases per year), 35\% have CRNAs (65\% physicians only) and for larger programs (>750 cases per year), one-third use CRNAs (two-thirds, physicians only).

Table 1: Cardiac surgery program size (major cardiac cases/year)

\begin{tabular}{cl} 
Major Cases (per year) & Respondents $(\mathrm{n}=40)$ \\
\hline $51-100$ & $2(5 \%)$ \\
$101-150$ & $3(7.5 \%)$ \\
$151-200$ & $10(25 \%)$ \\
$201-250$ & $3(7.5 \%)$ \\
$251-300$ & $2(5 \%)$ \\
$301-400$ & $1(2.5 \%)$ \\
$401-500$ & $5(12.5 \%)$ \\
$501-750$ & $1(2.5 \%)$ \\
$>750$ & $13(32.5 \%)$
\end{tabular}

For smaller programs, only 1 of $15(6.7 \%)$ programs that responded have a formal cardiothoracic residency or fellowship training program. However, $50 \%$ of medium sized programs (201-749 cases, $n=12)$ reported having a training program while $77 \%(n=10 / 13)$ of larger programs have training programs with residents or fellows involved in cardiac surgery cases.

Table 2 illustrates the spectrum of who is involved in the management of patients and characteristics that help describe the nature and complexity of the specific programs (i.e. whether a program performs ventricular assist devices, transplants, catheter-based structural heart interventions). Table 2 also outlines whether various types of trainees are involved in the intraoperative care of cardiac surgery patients, employment status of anesthesia providers, and country of origin.

Table 2: Intra-operative management:

$\begin{array}{ll}\text { Dedicated Cardiothoracic Anesthesiologist (MD/DO) }{ }^{+} & 28(70 \%) \\ \text { Use of CRNAs } & \\ \text { Yes } & 15(37.5 \%) \\ \quad \text { No - MD/DO Attendings only } & 16(40 \%) \\ \text { No - MD/DO Attendings with anesthesia residents } & 9(22.5 \%) \\ \text { Intraoperative TEE } & \\ \quad \text { All cardiac cases } & 30(75 \%) \\ \text { Only valves and intra-cardiac cases } & 7(17.5 \%) \\ \text { Only valves, intra-cardiac cases, also high-risk CABG only* } & 3(7.5 \%) \\ \text { Who performs TEE } & \\ >50 \% \text { of time, Anesthesia Team } & 5(12.5 \%) \\ >80 \% \text { of time, Anesthesia Team } & 6(15 \%) \\ \text { All performed by Anesthesia } & 23(57.5 \%) \\ \text { All performed by Cardiologist } & 5(12.5 \%)\end{array}$

\section{Training Program}


CRNA

General Surgery Residency

Cardiothoracic Surgery Fellowship

Anesthesia Residency

Cardiothoracic Anesthesia Fellowship

Cardiology Fellowship

Medical Students only

Multiple training programs

No training programs

\section{LVAD/Transplant Program}

No

No but developing

Bridge only

DT only

DT/Bridge

LVAD and Transplant

Percutaneous Valve (TAVR) Program

No

Developing

$>20$ implants/yr

$>50$ implants/yr

\section{Anesthesia hospital employed}

No

CRNA only employed

Physician only employed

CRNA and MD

\section{Country of response:}

USA

India

Argentina

Australia

UK

Italy

Israel

Canada

Russia

Saudi Arabia
$7(17.5 \%)$

$11(27.5 \%)$

$9(22.5 \%)$

$3(7.5 \%)$

$5(12.5 \%)$

$2(5 \%)$

$1(2.5 \%)$

$12(30 \%)$

$17(42.5 \%)$

$21(52.5 \%)$

2 (5\%)

$3(7.5 \%)$

2 (5\%)

4 (10\%)

5 (12.5\%)

$20(50 \%)$

4 (10\%)

7 (17.5\%)

9 (22.5\%)

15 (37.5\%)

4 (10\%)

5 (12.5\%)

15 (37.5\%)

27 (67.5\%)

$4(10 \%)$

2 (5\%)

1 (2.5\%)

1 (2.5\%)

$1(2.5 \%)$

1 (2.5\%)

1 (2.5\%)

1 (2.5\%)

$1(2.5 \%)$

Table Legend: Adult Cases: estimated number of major cardiac surgical procedures performed annually requiring cardiopulmonary bypass or off-pump techniques (i.e. off-pump coronary artery bypass); CABG: Coronary artery bypass grafting surgery; MD/DO: Medical or Osteopathic Physicians; TEE: Transesophageal Echocardiography; CRNA: Certified Registered Nurse Anesthetists; LVAD/Transplant Program: Program performs implantation of left ventricular assist devices (LVAD) and/or cardiothoracic transplantation; DT: Destination Therapy LVAD implantation; Bridge: LVAD bridge to transplant; TAVI: Trans-aortic valve implantation.

+ Dedicated Cardiothoracic Anesthesiologist was defined as an anesthesiologist with advanced, fellowship and/or specialty training in cardiothoracic surgery.

* High-risk CABG cases as defined by local Team providers on a case-by-case basis. 


\section{Discussion}

The analysis of the data supplied from the programs show variability across every dimension, even when grouped by similar size, and by teaching/non-teaching programs of the same size, despite all United States programs are held to the same standard in terms of their outcomes by Society of Thoracic Surgery (STS) reporting standards. Furthermore, there is growing expectation that such outcomes be publicly reported and potentially available for hospital reimbursements and patient education and decision making to assist in patient risk assessment.

The implications of this survey carry important implications. There are multiple studies that suggest that case volumes of programs impact outcomes, particularly in valve surgeries. This is leading to discussions and reimbursement hurdles that in the future may prevent certain small volume programs access to advanced technologies such as catheter-based or hybrid structural procedures. In addition, the increasing case complexity in cardiac surgery involves longer, more tedious cases, in which the use handoffs can prevent morbidity produced as a side-effect of provider fatigue [2]. While the primary attending surgeon rarely changes during the procedure, however, other members of the operative team changes are not uncommon, driven by resident duty hour restrictions [3], and the duty hours of other staff and team members. Evidence clearly exists that anesthesia handoffs intra-operatively adversely affect both morbidity and mortality in cardiac surgery patients [4]. However, the frequency of such events, the provider make-ups of the Team, and specific models of care remain poorly defined. As demonstrated by our brief survey, there is, in addition, large variability in the composition and functioning of the anesthesia-providing team. The anesthesia team is an important driver of cardiac/cardiac surgical outcomes.

While the STS has incorporated an anesthesia-specific component of their data collection system, few questions deal specifically with the care model structure and function. In order to reduce operative mistakes, preoperative checklists and team briefings have been shown to reduce communication errors, especially with multidisciplinary teams that include nurses, surgeons, anesthesiologists, and residents [5]. A similar system has yet to be developed for intra-operative handoffs.

Our data opens the question of team experience with one another, the use of the anesthesia providers, and what type of anesthesia providers used in cardiac surgery/procedures and what relationship this has to outcomes. In addition, the question of the role of other team members scrub techs, scrub nurses and circulating nurses to the outcomes could be another variable requiring consideration and study. I(https://www.sts.org/sites/default/files/documents/STS-AdultAnesthesia SampleDAR Blank.pdf).

Given the programmatic implications to programs and clinical, our study would suggest that the exploration of these factors deserve more study in the quest to improve patient outcomes, and provide guidance to the teams most effective in achieving this goal.

\section{Limitations}

The major limitation of this study is the small number of reported cases - and specifically, the low number of responses from programs outside of the United States. While the limited number of responses, combined with the inherent variabilities as reported makes it difficult to draw clearly defined conclusions, our findings do support the hypothesis that there are large (and potentially significant - depending on how defined) variabilities in how "anesthesia" care is delivered to patients undergoing cardiac surgery.

The other major limitation in this study is the assumption that variabilities in anesthesia care models potentially have an impact on patient outcomes. While there is a natural assumption that the surgeon has the largest impact on a patient outcome, particularly in the context of the technical aspects of the procedure (quality of anastomosis, myocardial protection, tissue trauma, response to unusual and/or unexpected intra-operative findings, etc) there is literature to suggest that the role of a surgeon, regardless of experience, is only one variable impacting patient outcomes [6]. In fact, there is growing evidence to suggest that the operating surgeon has little role in preventing most 
adverse outcomes after cardiac surgery [7]. As such, it is imperative to better understand those other factors that impact outcomes - hence, our hypothesis that variabilities exist in anesthesia care delivery models. This hypothesis, and our findings, hopefully will lay the foundation for exploring the relationships between different models of care - and potentially even different providers or types of providers - and their impact on patient post-operative outcomes. Again, while the technical expertise and judgment of the surgeon has an obvious role in outcomes, it is a reasonable assumption that similar experiences, cognitive and technical interventions, training and responses to intra-operative events by members of the anesthesia team also have an impact. Defining the magnitude of that impact and opportunities for improvement should be explored. As mentioned above, these concepts serve as the foundation for the development of a joint database between the Society of Cardiovascular Anesthesiologists and the Society of Thoracic Surgeons [8].

\section{Conclusions}

The operating room in cardiothoracic surgery is a dynamic environment, often with changes in staff throughout the surgery due to multiple factors. Anesthesia is a key member of the team with significant influence on outcomes for the patient. Our study shows that there is great variability, across every dimension of the international CT programs in regards to anesthesia in the role and staffing of anesthesia providers. Given the importance of the anesthesia team on patient outcomes, this study would suggest the opportunity for further investigation to determine the influence of different models on patient outcomes, to help define best practices.

Author Contributions: All authors contributed to the initial hypothesis, methods design, data analysis, and writing and reviewing of the manuscript. All authors have read and agreed to the published version of the manuscript and have contributed substantially to the work reported.

Funding: No additional or external funding supported this work.

Acknowledgments: None

Conflicts of Interest: The authors declare no conflicts of interest

\section{References}

1. Jones PM, Cherry RA, Allen BN, Jenkyn KM, Shariff SZ, Flier S, Vogt KN, Wijeysundera DN. Association between handover of anesthesia care and adverse postoperative outcomes among patients undergoing major surgery. JAMA. 2018 Jan 9;319(2):143-53.

2. Warltier DC, Howard SK, Rosekind MR, Katz JD, Berry AJ. Fatigue in Anesthesia: Implications and Strategies for Patient and Provider Safety. Anesthesiology: The Journal of the American Society of Anesthesiologists. 2002 Nov 1;97(5):1281-94.

3. Ahmed N, Devitt KS, Keshet I, Spicer J, Imrie K, Feldman L, Cools-Lartigue J, Kayssi A, Lipsman N, Elmi M, Kulkarni AV. A systematic review of the effects of resident duty hour restrictions in surgery: impact on resident wellness, training, and patient outcomes. Annals of Surgery. 2014; 259(6): 1041-1053.

4. Hudson CC, McDonald B, Hudson JK, Tran D, Boodhwani M. Impact of anesthesia handover on mortality and morbidity in cardiac surgery: a cohort study. Journal of Cardiothoracic \& Vascular Anesthesia. 2015; 29(1): 11-16.

5. Lingard L, Regehr G, Orser B, Reznick R, Baker GR, Doran D, Espin S, Bohnen J, Whyte S. Evaluation of a preoperative checklist and team briefing among surgeons, nurses, and anesthesiologists to reduce failures in communication. Archives of Surgery. 2008; 143(1): 12-17.

6. Edwards FH, Ferraris VA, Kurlansky PA, Lobdell KW, He X, O’Brien SM, Furnary AP, Rankin JS, Vassileva CM, Fazzalari FL, Magee MJ. Failure to rescue rates after coronary artery bypass grafting: an analysis from the Society of Thoracic Surgeons adult cardiac surgery database. The Annals of thoracic surgery. 2016 Aug 1;102(2):458-64. 
7. Westaby S, De Silva R, Petrou M, Bond S, Taggart D. Surgeon-specific mortality data disguise wider failings in delivery of safe surgical services. European Journal of Cardio-Thoracic Surgery. 2015 Feb 1;47(2):341-5.

8. Del Rio JM, Abernathy III JJ, Taylor MA, Habib RH, Fernandez FG, Bollen BA, Lauer RE, Nussmeier NA, Glance LG, Petty III JV, Mackensen GB. The Adult Cardiac Anesthesiology Section of STS Adult Cardiac Surgery Database: 2020 Update on Quality and Outcomes. Anesthesia \& Analgesia. 2020 Nov 1;131(5):138396. 